\title{
Molecular Typing of Clinical and Environmental Cryptococcus neoformans Strains Isolated in Italy
}

\author{
Gabriella Pini*, Elisabetta Faggi, Eleonora Bravetti \\ Department of Experimental and Clinical Medicine, University of Florence, Florence, Italy \\ Email: ^g.pini@unifi.it
}

How to cite this paper: Pini, G., Faggi, E. and Bravetti, E. (2017) Molecular Typing of Clinical and Environmental Cryptococcus neoformans Strains Isolated in Italy. Open Journal of Medical Microbiology, 7, 77-85. https://doi.org/10.4236/ojmm.2017.74007

Received: November 2, 2017

Accepted: December 25, 2017

Published: December 28, 2017

Copyright @ 2017 by authors and Scientific Research Publishing Inc. This work is licensed under the Creative Commons Attribution International License (CC BY 4.0).

http://creativecommons.org/licenses/by/4.0/ (c) (i) Open Access

\begin{abstract}
Cryptococcus neoformans is an encapsulated yeast causing mainly opportunistic infections. DNA molecular typing techniques divided $C$. neoformans into four major molecular types (AFLP1/VNI, AFLP1A/VNB/VNII, AFLP1B/ VNII, AFLP3/VNIII, AFLP2/VNIV) characterized by different pathogenicity, geographical distribution and susceptibility to antifungal treatments. In this study 170 Italian $C$. neoformans clinical isolates (CI) and 32 environmental isolates (EI), collected and serotyped during a ten-year period (1985 to 1995), were genotyped using $[\mathrm{GACA}]_{4}$ microsatellite PCR fingerprinting. The molecular types were compared to their geographic distribution, specimen sources and patient's risk factors. All four molecular types were described among the $\mathrm{CI}$ and only VNI and VNIV among the EI. VNIV molecular type was isolated with significant prevalence among the $\mathrm{CI}$ and VNI among the EI. A different geographical distribution of molecular types was detected: VNIV was the most prevalent in the North and Center of Italy; VNIII was isolated almost exclusively in the Center. No significant correlation among molecular types versus predisposing diseases or isolation sources was detected. The strains isolated from different body sites of the same patient (17 cases) were of the same genotype. Five out 9 cases relapsed with a different molecular type. This preliminary investigation shows a high intraspecies variability and reveals a nonhomogeneous distribution of $C$. neoformans molecular types in Italy.
\end{abstract}

\section{Keywords}

Cryptococcus neoformans, Clinical Isolates, Environmental Isolates, Molecular Typing, Molecular Types Distribution

\section{Introduction}

The genus Cryptococcus comprises more than 70 basidiomycetous fungal spe- 
cies characterized as variously encapsulated budding yeasts, of which mostly Cryptococcus neoformans and Cryptococcus gattii have been associated with life-threatening infections in humans and other animals, while infections with other species have rarely been reported [1]. Recently, a new taxonomic proposal splits $C$. neoformans into two species (C. neoformans and Cryptococcus deneoformans) and C. gattii into a total of five species (C. gattii, Cryptococcus bacillisporus, Cryptococcus deuterogattii, Cryptococcus tetragattii and Cryptococcus decagattii) [2]. Since this is still under discussion [3], in this study we continue to adopt the classical taxonomy which classifies the agents of cryptococcosis into two species, $C$. neoformans and $C$. gattii. $C$. neoformans is classified into two varieties and three serotypes: $C$. neoformans var. grubii (serotype A), C. neoformans var. neoformans (serotype $\mathrm{D}$ ) and the hybrid serotype $\mathrm{AD}$, whereas $C$. gattii comprises two serotypes, B and C [4].

Several molecular typing methods have been used for studying the molecular epidemiology of $C$. neoformans/ $C$. gattii species complex. The most common approaches include amplified fragment length polymorphism (AFLP) analysis [5], PCR fingerprinting [6], PCR-restriction fragment length polymorphism (RFLP) analysis [7] as well as multi locus sequence typing (MLST) [8]. DNA molecular typing techniques divide $C$. neoformans into four major molecular types (AFLP1/VNI, AFLP1A/VNB/VNII, AFLP1B/VNII, AFLP3/VNIII, AFLP2/VNIV) characterized by different pathogenicity, geographical distribution and susceptibility to antifungal treatments [9] [10]. C. neoformans var. grubii (serotype A/VNI genotype) has a worldwide distribution and is responsible for more than $80 \%$ of cryptococcal diseases [11] [12], whereas C. neoformans var. neoformans (serotype D/VNIV genotype) is found predominantly in Western Europe [9] [13] and South America [12] [14]. In addition, in the Mediterranean area of Europe, a higher prevalence of $C$. neoformans serotype AD hybrids (VNIII genotype) has been reported [15] [16].

In this study we investigated the genetic diversity of 202 clinical and environmental C. neoformans isolates, collected during the 1985-1995 period. The clinical strains were previously characterized [17] [18] but a comprehensive genotypic analysis of all collected $C$. neoformans strains has not been performed. For this purpose a preliminary genomic characterization, using the microsatellite PCR genotyping technique, was performed to assess the genetic diversity among Italian C. neoformans strains isolated in the early years of the AIDS outbreak.

\section{Materials and Methods}

One hundred and seventy Italian C. neoformans clinical isolates (CI) and 32 environmental isolates (EI), collected from 1985 to 1995 and stored in the Microbiology laboratory of Florence University, were typed by PCR fingerprinting. At the time of isolation, all the strains were identified by classical methods based on the urease production on Christensen's Urea Agar, the presence of a capsule, the melanin production and the assimilation of nitrogen and carbon sources [19]. 
To distinguish between $C$. neoformans and $C$. gattii, culture on canavanine-glycinebromothymol blue (CGB) medium [20] and growth in the medium containing d-proline [21] have been performed. In addition, all strains identification were also performed using the ID32C system (BioMérieux AS, Marcy l'Etoile, France). Slide agglutination test with specific monoclonal antibodies for capsular polysaccharide Crypto-check kit (Iatron Laboratories Inc., Tokio, Japan) was used for serotyping. The human strains, 77 serotype A and 93 serotype D, were isolated from 134 patients, hospitalized in 21 different cities, mainly in northern and central Italy. From seventeen patients, two or more specimens were collected from different body sites. Nine patients had relapses of cryptococcal infections defined by a period between two positive cultures of more than 120 days. Pre-existing condition in patients were HIV/AIDS ( $\mathrm{n}=121,90.3 \%)$, non-HIV/ AIDS-related immunodeficiency ( $\mathrm{n}=7,5.2 \%$ ) (e.g., immunosuppressive therapy or organ transplantation), immunocompetent $(\mathrm{n}=2,1.5 \%)$, and "unknown" for 4 patients (2.9\%). The CI were mainly from liquor $(n=110,65 \%)$, blood $(n=26$, $15 \%)$ and the $20 \%(\mathrm{n}=34)$ from other clinical specimens (i.e. urine, skin, sputum, prostatic secretions). Environmental isolates were from soil $(\mathrm{n}=14)$ and bird dried excreta $(n=18)$, mostly from pigeons, sampled in the north $(n=16)$, center $(n=2)$ and south $(n=14)$ of Italy. Twenty-two were serotype A and 10 serotype $\mathrm{D}$.

Each isolate was stored at room temperature in sterile distilled water and annually checked. Prior to the molecular biological analysis, the strains were revived and checked for purity by sub culturing on Sabouraud dextrose agar (DifcoBecton, Dickinson and Company, Milan, Italy) at $37^{\circ} \mathrm{C}$ for 7 days.

The following set of laboratory standard reference strains, representing each of the eight major molecular types of the $C$. neoformans/ $C$. gattii species complex were used: WM148 (serotype A, VNI), WM626 (serotype A, VNII), WM628 (serotype AD, VNIII), WM629 (serotype D, VNIV), WM179 (serotype B, VGI), WM178 (serotype B, VGII), WM161 (serotype B, VGIII), and WM779 (serotype C, VGIV) [6].

For this study, the strains were cultured on Sabouraud dextrose agar at $37^{\circ} \mathrm{C}$ for $48 \mathrm{~h}$ and the phenol-chloroform DNA extraction was performed as previously described [22]. The microsatellite-specific primer $[\mathrm{GACA}]_{4}$ (5' GAC AGACAGACAGACA 3') was used as a single primer in the PCR reactions, carried out in a final volume of $50 \mu$ l. Each reaction tube contained $1 \times$ PCR buffer (10 mM Tri- $\mathrm{HCl} \mathrm{pH} 8.3,50 \mathrm{mM} \mathrm{HCl}, 1.5 \mathrm{mM} \mathrm{MgCl}_{2}$ ) (Applied Biosystems, Foster City CA, USA), 3 mM MgAcetate (Sigma Chemical Co. St. Louis, MO, USA), $200 \mu \mathrm{M}$ each of dATP, dTTP, dCTP, dGTP (Boehringer, Mannheim, Germany), 2.5 U AmpliTaq DNA polymerase (Applied Biosystems, Foster City CA, USA), $160 \mathrm{ng}$ primer [GACA] ${ }_{4}, 25 \mathrm{ng}$ DNA. PCR was performed for 35 cycles: denaturation $94^{\circ} \mathrm{C}$ for 20 second, annealing $50^{\circ} \mathrm{C}$ for 1 minute, extension $72^{\circ} \mathrm{C}$ for 20 seconds, followed by a final extension cycle for 6 minutes at $72^{\circ} \mathrm{C}$ [6]. All PCR reactions were carried out in a thermal cycler (2720 Applied Biosystems, Foster City CA, USA) and each isolate was amplified in duplicate. Am- 
plification products were electrophoresed on $1.4 \%$ agarose gel at $60 \mathrm{~V}$ for $14 \mathrm{~cm}$. After a complete run, banding patterns were assigned visually by comparison with the molecular weight marker standard VI (Roche Diagnostics Germany).

The molecular types were assigned by comparison to the standard molecular type strains [6] (Figure 1). Only unambiguous and reproducible bands were included in the analysis.

For the statistical analysis, percentages were compared using the chi-square or the Fisher's exact test. A p-value of $\leq 0.05$ was considered statistically significant. The possible correlation between the molecular types of the tested isolates versus predisposing disease or isolation sources was investigated using Pearson's and Spearman's correlation coefficient.

\section{Results}

All four molecular types were described among CI and only VNI and VNIV among EI. Overall serotype A belong to VNI molecular type $(n=69)$, VNII ( $n=$ $2)$, VNIII $(\mathrm{n}=20)$ and VNIV $(\mathrm{n}=8)$. Serotype D is associated mostly with VNIV molecular type $(\mathrm{n}=101)$ and very few isolates with VNIII $(\mathrm{n}=1)$ and VNI $(\mathrm{n}=1)$. VNIV molecular type was isolated with significant prevalence among CI $(\mathrm{p}<0.01)$ and VNI among the EI (Table 1).

The VNIV molecular type was the most prevalent in the North and Center of Italy (69\% and 49\% respectively). The VNIII molecular type was isolated mainly in the Center of Italy $(20 \%)$ with a significant prevalence $(\mathrm{p}<0.05)$ (Table 2$)$.

Both environmental and clinical strains isolated in Southern Italy were VNI genotype, except for one VNIV clinical isolate.

Most of the isolates were from liquor of AIDS patients. From this group of patients all four molecular types have been described (Table 3).

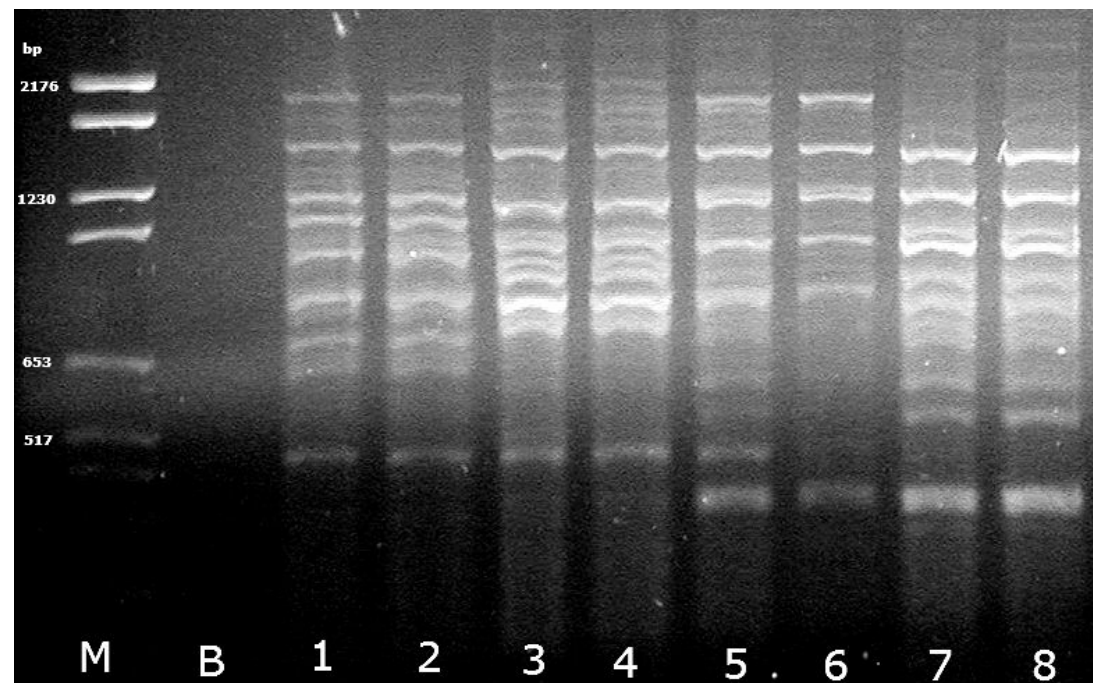

M: molecular weight marker standard, B: control without DNA, 1 - 2: VNI molecular type, 3 - 4: VNII molecular type, 5 - 6: VNIII molecular type, 7 - 8: VNIV molecular type

Figure 1. Electrophoretic separation of PCR fingerprinting products obtained by amplifying $C$. neoformans DNA with the single primer (GACA) . 
Table 1. Genotyping by (GACA) ${ }_{4}$ PCR fingerprinting of Cryptococcus neoformans clinical and environmental isolates.

\begin{tabular}{ccccccc}
\hline \multirow{2}{*}{ Genotype } & \multicolumn{3}{c}{ Clinical isolates N (\%) } & \multicolumn{3}{c}{ Environmental isolates N (\%) } \\
\cline { 2 - 7 } & Serotype A & Serotype D & Total & Serotype A & Serotype D & Total \\
\hline VNI & 47 & 1 & $48(28 \%)$ & 22 & 0 & $22(69 \%)^{\mathrm{c}}$ \\
VNII & 2 & 0 & $2(1 \%)$ & 0 & 0 & 0 \\
VNIII & 20 & 1 & $21(12 \%)$ & 0 & 0 & 0 \\
VNIV & 8 & 91 & $99(58 \%)^{\mathrm{a}}$ & 0 & 10 & $10(31 \%)$ \\
Total & $77(45 \%)$ & $93(55 \%)$ & 170 & $22(69 \%)^{\mathrm{b}}$ & $10(31 \%)$ & 32 \\
\hline
\end{tabular}

a. $\mathrm{p}<0.01$, b. $\mathrm{p}<0.05, \mathrm{c} . \mathrm{p}<0.01$.

Table 2. Geographic distribution of the Cryptococcus neoformans molecular types.

\begin{tabular}{ccccccc}
\hline \multirow{2}{*}{ Genotype } & \multicolumn{3}{c}{ Clinical isolates N (\%) } & \multicolumn{2}{c}{ Environmental isolates N (\%) } \\
\cline { 2 - 7 } & North & Center & South & North & Center & South \\
\hline VNI & $23(24 \%)$ & $19(28 \%)$ & $6(86 \%)$ & $8(50 \%)$ & 0 & $14(100 \%)$ \\
VNII & 0 & $2(3 \%)$ & 0 & 0 & 0 & 0 \\
VNIII & $7(7 \%)$ & $14(20 \%)^{\mathrm{a}}$ & 0 & 0 & 0 & 0 \\
VNIV & $65(69 \%)$ & $33(49 \%)$ & $1(14 \%)$ & $8(50 \%)$ & $2(100 \%)$ & 0 \\
Total & 95 & 68 & 7 & 16 & 2 & 14 \\
\hline
\end{tabular}

a. $\mathrm{p}<0.05$

Table 3. Distribution of the molecular types according to the underlying disease and the specimen sources of the Clinical Isolates.

\begin{tabular}{cccccccccc}
\hline & \multicolumn{3}{c}{ Disease } & \multicolumn{5}{c}{ Specimen sources } \\
\cline { 2 - 10 } Genotype & AIDS & Other immunodeficiency & No immunodeficiency & Unknown status & Liquor & Blood & Skin & $\begin{array}{c}\text { Outer } \\
\text { sources }\end{array}$ & $\begin{array}{c}\text { Unknown } \\
\text { sources }\end{array}$ \\
\hline VNI & 37 & $6^{\mathrm{a}}$ & 0 & 5 & 34 & 5 & 0 & 5 & 4 \\
VNII & 1 & 1 & 0 & 0 & 1 & 0 & 1 & 0 & 0 \\
VNIII & 20 & 1 & 0 & 0 & 15 & 4 & 0 & 2 & 0 \\
VNIV & 93 & 2 & 2 & 2 & 60 & 17 & 7 & 14 & 1 \\
Total & 151 & 10 & 2 & 7 & 110 & 26 & 8 & 21 & 5 \\
\hline
\end{tabular}

a. $\mathrm{p}<0.05$

Among the isolates from patients with immunodeficiency other than AIDS, VNI was significantly prevalent $(\mathrm{p}<0.05)$.

The less represented VNII molecular type were isolated from a single liquor sample of a leukemic patient relapsed and from a skin specimen of an AIDS patient, both in the central Italy.

The VNIII strains were isolated almost exclusively from patients with AIDS. The two isolates from immunocompetent patients showed VNIV molecular type.

No significant correlation among the molecular types versus pre-existing con- 
ditions or isolation sources was detected.

When specimens were obtained in a single patient from multiple body sites (n = 17), Cryptococcus isolates were from the same species and molecular type. Instead, the isolates collected during relapse presented a different molecular type from those from first infection in five cases out of nine. The shift more frequent was from VNIV to VNIII or conversely.

\section{Discussion}

Worldwide C. neoformans var. grubii, VNI, is the cause of more than $80 \%$ of all cryptococcal infections, mostly in immunocompromised patients [12]. In contrast, our results revealed that VNIV molecular type was the most prevalent species causing infection (58\%) in the population considered in this study, especially in AIDS patients. In addition, VNIV prevalence resulted higher than in others European and Italian reports (14\% - 35\%) [16] [23] [24] [25].

Previous studies [26] reported a higher prevalence of $C$. neoformans. var. grubii (VNI) in the south of Italy, while $C$. neoformans var. neoformans (VNIV) seems more common in the north [16] [24]. Our results support the same trend of data. Indeed, Cryptococcus molecular types were not homogeneously distributed among Italy with a clear prevalence of VNIV in the north, while VNI prevailed in the south, both among the $\mathrm{CI}$ that among the EI. This might reflect a different susceptibility to temperature [27]. The VNIII molecular type was found with a significantly higher percentage in central Italy than in the north (20 and $7 \%$ respectively) and it was not found in the south. In the center of Italy the two haploid forms (VNI and VNIV) were found in similar percentages and this might have facilitated the emergence of hybrid strains.

The only two isolates VNII genotype recovered in this study confirms the rare distribution in Italy [28] and worldwide [10] [12] [15].

Classically serotype A is associated to VNI and VNII molecular type, and serotype D to VNIV. Discrepancies between serotype and molecular type were observed in this study as 8 serotype A isolates were found belonging to VNIV molecular type and one serotype D was found belonging to VNI. This is possible due to the limit of serotyping method performed in the ' 90 , compared with PCR fingerprinting, combined with the high presence of hybrids in Europe [16] [29] which can differently express serotype. In our investigation, most of the VNIII isolates were previously considered to be of serotype A (95\%), probably because some hybrid forms of $C$. neoformans only show one serotype as reported in other studies [15] [24] [29].

In this study VNI prevalence was significantly higher among non-HIV/AIDS patients compared to AIDS patients. Molecular type VNI is known to be an important agent of cryptococcal meningitis in both immunocompromised and immunocompetent patients in Italy [28]. In addition, these results are in agreement with studies from Korea and Japan that identified a clonal population, designated genotype VNIc, commonly isolated from non-AIDS patients [30] 
[31].

All the isolates from the skin were VNIV molecular type, excluded one VNII isolate, both from immunocompetent and AIDS patients. This finding confirmed the association of serotype D/VNIV molecular type with cutaneous lesions reported in previous studies [24] [32]. The dermatotropism associated with serotype D/VNIV molecular type strains can be caused by the greater thermal susceptibility in infected tissues and it may reflect a preference of VNIV molecular type for growth in the cooler tissues of the skin [27].

The reports of multiple infections caused by strains with different genotypes are increased [10] [33]. Desnoss-Olliver et al. [29] found in France a high proportion (21.5\%) of proven or probable infections due to a mixture of genotypes, serotypes and/or ploidies of $C$. neoformans strains. In our research, the strains isolates during Cryptococcus infections were characterized by a single molecular type, while the relapses were mostly caused by molecular types different from the ones described in the previous infection, suggesting a new acquired infection.

The (GACA) ${ }_{4}$-PCR fingerprinting typing method provided interesting results but further genomic typing investigations, such as multi locus sequence typing technique, are needed to better understand the molecular epidemiology of cryptococcosis in Italy in the early years of the AIDS outbreak.

\section{References}

[1] Khawcharoenporn, T., Apisarnthanarak, A. and Mundy, L.M. (2007) Non-neoformans Cryptococcal Infections: A Systematic Review. Infection, 35, 51-58. https://doi.org/10.1007/s15010-007-6142-8

[2] Hagen, F., Khayhan, K., Theelen, B., Kolecka, A., Polacheck, I., et al. (2015) Recognition of Seven Species in the Cryptococcus gattii/Cryptococcus neoformans Species Complex. Fungal Genetics and Biology, 78, 16-48. https://doi.org/10.1016/j.fgb.2015.02.009

[3] Kwon-Chung, K.J., Bennett, J.E., Wickes, B.L., Meyer, W., Cuomo, C.A., et al. (2017) The Case for Adopting the "Species Complex" Nomenclature for the Etiologic Agents of Cryptococcosis. mSphere, 2, e00357-16. https://doi.org/10.1128/mSphere.00357-16

[4] Kwon-Chung, K.J. and Varma, A. (2006) Do Major Species Concepts Support One, Two or More Species within Cryptococcus neoformans? FEMS Yeast Research, 6, 574-587. https://doi.org/10.1111/j.1567-1364.2006.00088.x

[5] Boekhout, T., Theelen, B., Diaz, M., Fell, J.W., Hop, W.C., et al. (2001) Hybrid Genotypes in the Pathogenic Yeast Cryptococcus neoformans. Microbiology, 147, 891-907. https://doi.org/10.1099/00221287-147-4-891

[6] Meyer, W., Marszewska, K., Amirmostođan, M., Igreja, R.P., Hardtke, C., et al. (1999) Molecular Typing of Global Isolates of Cryptococcus neoformans var. neoformans by Polymerase Chain Reaction Fingerprinting and Randomly Amplified Polymorphic DNA-A Pilot Study to Standardize Techniques on Which to Base a Detailed Epidemiological Survey. Electrophoresis, 20, 1790-1799. https://doi.org/10.1002/(SICI)1522-2683(19990101)20:8<1790::AID-ELPS1790>3.0. CO;2-2 
[7] Meyer, W., Castañeda, A., Jackson, S., Huynh, M. and Castañeda, E., IberoAmerican Cryptococcal Study Group (2003) Molecular Typing of Iberoamerican Cryptococcus neoformans Isolates. Archive of Emerging Infectious Diseases, 9, 189-195. https://doi.org/10.3201/eid0902.020246

[8] Meyer, W., Aanensen, D.M., Boekhout, T., Cogliati, M., Diaz, M.R., et al. (2009) Consensus Multi-Locus Sequence Typing Scheme for Cryptococcus neoformans and Cryptococcus gattii. Medical Mycology, 47, 561-570. https://doi.org/10.1080/13693780902953886

[9] Cogliati, M. (2013) Global Molecular Epidemiology of Cryptococcus neoformans and Cryptococcus gattii: An Atlas of the Molecular Types. Scientifica, 2013, Article ID: 675213. https://doi.org/10.1155/2013/675213

[10] Hagen, F., Illnait-Zaragozí, M.T., Meis, J.F., Chew, W.H, Curfs-Breuker, I., et al. (2012) Extensive Genetic Diversity within the Dutch Clinical Cryptococcus neoformans Population. Journal of Clinical Microbiology, 50, 1918-1926. https://doi.org/10.1128/JCM.06750-11

[11] Antinori, S. (2013) New Insights into HIV/AIDS-Associated Cryptococcosis. ISRN AIDS, 2013, Article ID: 471363. https://doi.org/10.1155/2013/471363

[12] Meyer, W. and Trilles, L. (2010) Genotyping of the Cryptococcus neoformans/C. gattii Species Complex. Australian Biochemist, 41, 12-16.

[13] Cogliati, M., D’amicis, R., Zani, A., Montagna, M.T., Caggiano, G., et al. (2016) Environmental Distribution of Cryptococcus neoformans and C. gattii around the Mediterranean Basin. FEMS Yeast Research, 16, fow045.

https://doi.org/10.1093/femsyr/fow045

[14] Barreto de Oliveira, M.T., Boekhout, T., Theelen, B., Hagen, F., Baroni, F.A., et al. (2004) Cryptococcus neoformans Shows a Remarkable Genotypic Diversity in Brazil. Journal of Clinical Microbiology, 42, 1356-1359. https://doi.org/10.1128/JCM.42.3.1356-1359.2004

[15] Frasés, S., Ferrer, C., Sánchez, M. and Colom-Valiente, M.F. (2009) Molecular Epidemiology of Isolates of the Cryptococcus neoformans Species Complex from Spain. Revista Iberoamericana de Micología, 26, 112-117. https://doi.org/10.1016/S1130-1406(09)70021-X

[16] Viviani, M.A., Cogliati, M., Esposto, M.C., Lemmer, K., Tintelnot, K., et al. (2006) European Confederation of Medical Mycology (ECMM) Cryptococcosis Working Group. Molecular Analysis of 311 Cryptococcus neoformans Isolates from a 30-Month ECMM Survey of Cryptococcosis in Europe. FEMS Yeast Research, 6, 614-619. https://doi.org/10.1111/j.1567-1364.2006.00081.x

[17] Gargani, G. and Pini, G. (1998) Indagine Sulla Criptococcosi in Italia. 1. Osservazioni Epidemiologiche. Microbiologia Medica, 13, 648-655.

[18] Gargani, G. and Pini, G. (1998) Indagine Sulla Criptococcosi in Italia. 2. Caratteri Degli Stipiti Isolati. Microbiologia Medica, 13, 656-661.

[19] Reiss, E., Shadomy, H.J. and Lyon, G.M. (2011) Fundamental Medical Mycology. John Wiley \& Sons, Eds., Haboken, New Jersey, Chapter 12.13.

https://doi.org/10.1002/9781118101773

[20] Kwon-Chung, K.J., Polacheck, I. and Bennett, J.E. (1982) Improved Diagnostic Medium for Separation of Cryptococcus neoformans var. neoformans (Serotypes A and D) and Cryptococcus neoformans var. gattii (serotypes B and C). Journal of Clinical Microbiology, 15, 535-537.

[21] Dufait, R., Velho, R. and De Vroey, C. (2000) Rapid Identification of the Two Varieties of Cryptococcus neoformans by D-Proline Assimilation. Clinical Microbiol- 
ogy Reviews, 13, 122-143.

[22] Pini, G., Faggi, E., Campisi, E. and Gargani, G. (1998) Typing of Cryptococcus neoformans Strains by Random PCR. Journal de Mycologie Médicale, 8, 7-12.

[23] Sanchini, A., Smith, I.M., Sedlacek, L., Schwarz, R., Tintelnot, K., et al. (2014) Molecular Typing of Clinical Cryptococcus neoformans Isolates Collected in Germany from 2004 to 2010. Medical Microbiology and Immunology, 203, 333-340. https://doi.org/10.1007/s00430-014-0341-6

[24] FIMUA Cryptococcosis Network (2002) European Confederation of Medical Mycology (ECMM) Prospective Survey of Cryptococcosis: Report from Italy. Medical Mycology, 40, 507-517. https://doi.org/10.1080/mmy.40.5.507.517

[25] Cogliati, M., Tortorano, A.M. and FIMUA Cryptococcosis Network (2014) Second Prospective Survey on Cryptococcosis in Italy after 10 Years. Mycoses, 57, S33-S34.

[26] Pernice, I., Lo Passo, C., Criseo, G., Pernice, A. and Todaro-Luck, F. (1999) Molecular Subtyping of Clinical and Environmental Strains of Cryptococcus neoformans Variety Neoformans Serotype A Isolated from Southern Italy. Mycoses, 41, 117-124. https://doi.org/10.1111/j.1439-0507.1998.tb00312.x

[27] Martinez, L.R., Garcia-Rivera, J. and Casadevall, A. (2001) Cryptococcus neoformans var. neoformans (serotype D) Strains Are More Susceptible to Heat than $C$. neoformans var. grubii (serotype A) Strains. Journal of Clinical Microbiology, 39, 3365-3367. https://doi.org/10.1128/JCM.39.9.3365-3367.2001

[28] Cogliati, M., Zamfirova, R.R., Tortorano, A.M., Viviani, M.A. and FIMUA Cryptococcosis Network (2013) Molecular Epidemiology of Italian Clinical Cryptococcus neoformans var grubii Isolates. Medical Mycology, 51, 499-506. https://doi.org/10.3109/13693786.2012.751642

[29] Desnos-Ollivier, M., Patel, S., Raoux-Barbot, D., Heitman, J., Dromer, F., et al. (2015) Cryptococcosis Serotypes Impact Outcome and Provide Evidence of Cryptococcus neoformans Speciation. MBio, 6, e00311-15. https://doi.org/10.1128/mBio.00311-15

[30] Choi, Y.H., Ngamskulrungroj, P., Varma, A., Sionov, E., Hwang, S.M., et al. (2010) Prevalence of the VNIc Genotype of Cryptococcus neoformans in Non-HIV-Associated Cryptococcosis in the Republic of Korea. FEMS Yeast Research, 10, 769-778. https://doi.org/10.1111/j.1567-1364.2010.00648.x

[31] Mihara, T., Izumikawa, K., Saijo, T., Ngamskulrungroj, P., Umeyama, T., et al. (2012) Multilocus Sequence Typing of Cryptococcus neoformans in Non-HIV Associated Cryptococcosis in Nagasaki, Japan. Medical Mycology, 51, 252-260. https://doi.org/10.3109/13693786.2012.708883

[32] Naka, W., Masuda, M., Konohana, A., Shinoda, T. and Nishikawa, T. (1995) Primary Cutaneous Cryptococcosis and Cryptococcus neoformans Serotype D. Clinical and Experimental Dermatology, 20, 221-225. https://doi.org/10.1111/j.1365-2230.1995.tb01306.x

[33] Guinea, J., Hagen, F., Peláez, T., Boekhout, T., Tahoune, H., et al. (2010) Antifungal Susceptibility, Serotyping, and Genotyping of Clinical Cryptococcus neoformans Isolates Collected during 18 Years in a Single Institution in Madrid, Spain. Medical Mycology, 48, 942-948. https://doi.org/10.3109/13693781003690067 